\title{
Estrategia didáctica para el desarrollo del aprendizaje autónomo en estudiantes de la carrera de Derecho
}

\author{
Didactic strategy for the development of autonomous learning in law \\ students
}

\section{Lourdes Rivas Zapata \\ lulita_rulitos_@hotmail.com \\ Universidad Domingo Savio Potosí, Bolivia}

\section{RESUMEN}

El presente artículo tiene como propósito compartir una estrategia didáctica para fomentar el aprendizaje autónomo en los estudiantes. Como metodología de construcción de la investigación se utilizó el método analíticosintético; el método inductivo-deductivo; el enfoque sistémico; y el método análisis documental. Con ello se determinan los principales referentes teóricos, del proceso de enseñanza aprendizaje, aprendizaje autónomo, y como desarrollar habilidades para lograr un aprendizaje autónomo, caracterizando el estado actual del aprendizaje autónomo en el escenario estudiado. Se puede concluir que el proceso enseñanza aprendizaje resulta ser ampliamente dinámico y complejo, que los maestros deben ser ampliamente creativos para motivar a los estudiantes y desarrollar en ellos habilidades relacionadas al aprendizaje autónomo, pero que además estimulé el pensamiento crítico, creativo colaborativo y desarrollé las habilidades cognitivas y meta cognitivas del estudiante para ser creadores de conocimiento.

Palabras clave: Estrategia didáctica; aprendizaje autónomo; carrera de Derecho; Universidad Domingo Savio de Potosí

\section{ABSTRACT}

The purpose of this article is to share a didactic strategy to promote autonomous learning in students. The analytical-synthetic method was used as the research construction methodology; the inductive-deductive method; the systemic approach; and the documentary analysis method. This determines the main theoretical references, the teachinglearning process, autonomous learning, and how to develop skills to achieve autonomous learning, characterizing the current state of autonomous learning in the studied scenario. It can be concluded that the teaching-learning process turns out to be widely dynamic and complex, that teachers must be widely creative to motivate students and develop skills related to autonomous learning, but also stimulate critical, creative collaborative thinking and develop Cognitive skills and cognitive goal of the student to be knowledge creators.

Key words: didactic strategy; Autonomous Learning; law career; Domingo Savio University of Potosí 


\section{INTRODUCCIÓN}

En el actual y fluctuante mundo laboral cada vez más, es un requerimiento que los egresados de las universidades tengan una formación integral, conocimientos sólidos y una disciplina de trabajo que les permita continuar aprendiendo todo aquello que necesiten de acuerdo con los objetivos y retos que tengan que enfrentar a lo largo de su vida personal y profesional. Lo anterior se va formando desde edades tempranas, pero se consolida con los estudios universitarios, por ello, es necesario modelar y crear procesos de gestión de calidad en las instituciones universitarias, las cuales juegas un papel esencial en el proceso de enseñanza $y$ aprendizaje, dirigido y coordinado por el docente en el aula, el cual debe propiciar estrategias didácticas para que el estudiante aprenda de manera autónoma y autorregulada.

El aprendizaje autónomo constituye un reto, tanto para el docente como para el estudiante en su formación profesional, por ello, el presente artículo busca dar respuesta al problema científico de cómo estimular el aprendizaje autónomo en estudiantes de la carrera de derecho de la Universidad Privada Domingo Savio de Potosí. A medida en que pasan por las instituciones educativas, los modelos academicistas que aún predominan y que están centrados en la transmisión de contenidos por parte del docente y menos centrados en la elaboración personal y profesional del sujeto que aprende, van creando aprendices cada vez más dependientes, lo cual limita su aprendizaje autónomo.

Por ello, se hace necesario considerar como situación problemática la insuficiente gestión de aprendizaje autónomo por parte del estudiante durante el proceso enseñanza aprendizaje y la insuficiente creación por el docente de situaciones de enseñanza y aprendizajes que estimulen el aprendizaje autónomo en los estudiantes.

El estudiante universitario debe ser capaz de auto dirigir y autorregular el proceso mismo de adquisición de nuevos conocimientos, habilidades, mediante el estudio de diversos contenidos y de la experiencia misma que va adquiriendo, debe ser capaz de participar en actividades de aprendizaje que estimulen su inteligencia, su autodisciplina, que desarrollen actitudes y habilidades investigativas para formular $\mathrm{y}$ solucionar problemas de manera responsable e independiente, en fin el estudiante universitario debe estar capacitado para aprender a aprender y gestionar su propio proceso de aprendizaje. En la práctica las exigencias anteriores no se producen, en los estudiantes de primer semestre de la carrera de Derecho de la Universidad Privada Domingo Savio de Potosí (UPDS) se observa una tendencia hacia un aprendizaje memorístico, dependiente y reproductivo.

Se sabe que los conocimientos avanzan a una velocidad impresionante y no es posible que se implementen en la universidad planes de estudios que abarquen todo el contenido de una profesión, por lo que hay que darles a los estudiantes herramientas para que aprendan a aprender, a este factor, se suman los cambios tecnológicos constantes lo que exige tener universitarios autosuficientes en el tema aprendizaje, no solo durante la época universitaria, sino que se convierta en un tema estructural durante toda la vida.

Ante este panorama, se considera que la función del docente en la actualidad es orientar al estudiante para que desarrolle un conjunto de capacidades tanto intelectuales como socio-afectivas que le permitan continuar aprendiendo durante toda su vida y tomar conciencia de sí mismo, de sus motivaciones, capacidades y posibilidades. Se trata de que aprenda a trabajar con otros, 
pensar críticamente, resolver problemas, buscar la información necesaria, comprender la realidad desde distintas perspectivas y desde su complejidad, así como, tomar decisiones informadas y reflexionadas que le posibiliten asumir la responsabilidad de éstas $\mathrm{y}$ de las acciones que le siguen.

La educación en la actualidad requiere de profesionales capaces de resolver problemas principalmente a través del conocimiento; se deben hacer ajustes a la metodología que utilizan los docentes, debemos buscar medios para lograr mejorar estos procesos educativos, pues es un proceso precisamente hecho para lograr que el estudiante sea autónomo, pues el docente debe ser quien guie, además de fomentar el aprendizaje autónomo. Primero se le deberá dotar de herramientas al estudiante, mediante la utilización de estrategias que serán las que los motiven para lograr asimilar los contenidos y retener los mismos dentro del Proceso de Enseñanza Aprendizaje (PEA) y que su aprendizaje sea significativo.

Por todo ello, se plantea como interrogante fundamental ¿Cómo fomentar el aprendizaje autónomo en los estudiantes de primer semestre de la Carrera de Derecho de la Universidad Privada Domingo Savio?, esto plantea como objeto de estudio el aprendizaje autónomo en estudiantes universitarios, teniendo como campo de acción el proceso enseñanza aprendizaje, en estudiantes de primer semestre de la Carrera de Derecho de la Universidad Privada Domingo Savio, y se pretende proponer una estrategia didáctica para fomentar el aprendizaje autónomo en los estudiantes de primer semestre de la Carrera de Derecho de la Universidad Privada Domingo Savio.

Para responder estas preguntas se cumplieron las siguientes tareas: Determinación de los principales referentes teóricos, del proceso de enseñanza aprendizaje, aprendizaje autónomo, y como desarrollar habilidades para lograr un aprendizaje autónomo en estudiantes de primer semestre de la (UPDS); caracterización del estado actual del aprendizaje autónomo en los estudiantes de primer semestre de la carrera de Derecho de la Universidad Privada Domingo Savio; determinación de los componentes estructurales y su sistema de relaciones que se integran en una estrategia didáctica que fomenta el aprendizaje autónomo de los estudiantes de primer semestre de la carrera de Derecho de la Universidad Privada Domingo Savio. Y valoración de los resultados que se obtienen con la aplicación práctica de la estrategia didáctica que fomenta el aprendizaje autónomo en estudiantes de la carrera de Derecho de la Universidad Privada Domingo Savio.

\section{MÉTODO}

Para el desarrollo de la investigación, fueron utilizados en la investigación base de este a los métodos; analítico y sintético con la finalidad de profundizar en la teoría y obtener conclusiones y definiciones de conceptos de aprendizaje autónomo, para estructurar el marco teórico. Se utiliza además para el análisis de los datos obtenidos en el diagnóstico y la síntesis de las principales tendencias, también en la elaboración de la propuesta y en su valoración; el método inductivo-deductivo con la finalidad de particularizar solo en parte $y$ lograr generalizar los datos obtenidos de la muestra y llegar a una apreciación general del aprendizaje autónomo de los estudiantes de primer semestre de la carrera de Derecho de la Universidad Privada Domingo Savio, asimismo se utilizará, en la estructuración de la definición de la propuesta formulando los conceptos específicos y de esta forma dar solidez a la definición de aprendizaje 
autónomo y encontrar la estrategia didáctica de forma que sea adecuada a partir del tránsito de lo general a lo particular.

Igualmente se utilizó el enfoque sistémico, a todo lo largo de la investigación y además, es esencial en la estructuración de los componentes y su sistema de relaciones, en la estrategia didáctica, así como en la valoración de la misma que pasa por un proceso para llegar a su aplicación y el impacto que ella tiene en el fomento del aprendizaje autónomo en los estudiantes de primer semestre de la carrera de Derecho de la Universidad Privada Domingo Savio, lo cual está en correspondencia con las necesidades del campo de acción tratado.

Y por último el método análisis documental con la finalidad de analizar los principales documentos que están relacionados con el aprendizaje autónomo en los estudiantes de primer semestre de la carrera de Derecho de la Universidad Privada Domingo Savio. También los documentos que sustentan jurídicamente y orientan a los profesores sobre los procesos de enseñanza y aprendizaje.

Los instrumentos aplicados para obtener información necesaria sobre el aprendizaje autónomo fueron el cuestionario, que se aplicó a los docentes que fueron seleccionados del universo de la población de 19 docentes; los estudiantes fueron encuestados de una población de 150 estudiantes cuya muestra también fue de 150 estudiantes, es decir, fue censal; la guía documental y la entrevista.

\section{RESULTADOS Y DISCUSIÓN}

Estrategia didáctica para el desarrollo del aprendizaje autónomo en estudiantes del primer semestre de la carrera de derecho

Iniciemos esta parte retomando lo expuesto por Feo, (2009) quien plantea que las estrategias didácticas se pueden definir como los procedimientos o conjunto de ellos (métodos, técnicas, actividades) por los cuales el docente y los estudiantes organizan las acciones de manera consiente para construir y lograr metas previstas e imprevistas en el proceso enseñanza y aprendizaje, adaptándose a las necesidades de los participantes de manera significativa. Por su parte, Uria, (2001) define la estrategia didáctica como el análisis desde el trabajo del profesor, que es quien utiliza de manera consciente para promover el aprendizaje.

Para Brenes, (2003) la estrategia didáctica es aquel donde el educador puede utilizar y adecuar en el contexto escolar, muchos recursos valiosos para el descubrimiento, reflexión y de la integración de las actividades que promuevan el desarrollo físico, moral, social, cultural, además del intelectual, un ambiente agradable e interesante. El autor define que la estrategia didáctica se puede adecuar a diferentes actividades y promover otras en el proceso de enseñanza aprendizaje, para lo cual el docente elegirá las actividades más adecuadas que lleguen a favorecer el desarrollo físico, moral, cultural, intelectual y lograr un aprendizaje integral cumpliendo objetivos planificados.

De lo mencionado se puede decir que la estrategia didáctica es la forma como se desarrolla el proceso de enseñanza aprendizaje, conforme las necesidades que presenten los estudiantes, tanto docente como estudiante verán cómo mejorar este proceso de enseñanza aprendizaje y que este sea eficaz.

Ahora bien, el objetivo planteado por la autora fue elaborar una estrategia didáctica para el desarrollo del aprendizaje autónomo en estudiantes de primer semestre de la facultad de derecho de la UPDS. 
Primeramente, se verá cuáles son los componentes de la estrategia didáctica para luego pasar al desarrollo de los talleres, mapas conceptuales y debates; sesiones que irán dirigidas a los estudiantes de primer semestre de la UPDS y a través del contenido de la propuesta con la articulación de actividades de aprendizaje individual o grupal, proyectos, diálogos exposiciones para lograr que el estudiante pueda tener conocimiento sobre la materia que recibe; que el estudiante conozca sobre las estrategias de aprendizaje, y estilos de aprendizaje; que estudiante organicé su tiempo para resolver las tareas de su aprendizaje; fomentar la creatividad e innovación, sentido crítico y reflexivo en el estudiante; que el estudiante de una correcta utilización a las Tics y alcance sus objetivos a partir de los recursos disponibles.

\section{ESQUEMA DE LA PROPUESTA}

\section{ESTRATEGIA DIDÁTICA PARA EL DESARROLLO DEL APRENDIZAJE AUTÓNOMO DE ESTUDIANTES DE PRIMER SEMESTRE DE LA CARRERA DE DERECHO DE LA UPDS}

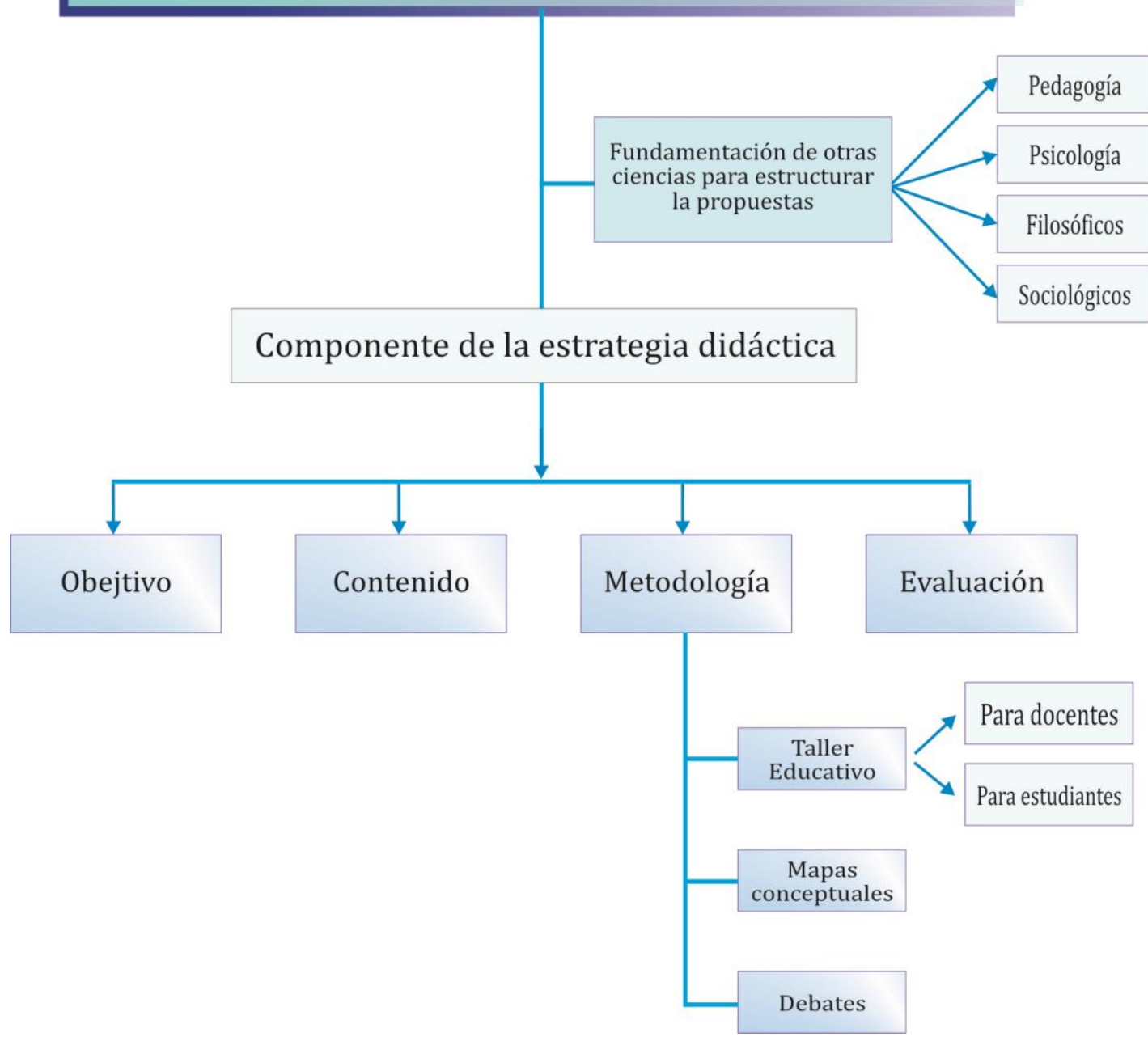




\section{Discusión}

Adentrarse en el estudio del proceso de enseñanza y aprendizaje requiere, referirse a otro proceso que se denomina proceso pedagógico, el cual posee un carácter más abarcador y general que está estrechamente relacionado con él. Al respecto Candau (2017) indica que el proceso de enseñanzaaprendizaje, que es un proceso pedagógico escolar que posee las características esenciales de éste, pero se distingue por ser mucho más sistemático, planificado, dirigido y específico por cuanto la interrelación maestro-estudiante, deviene en un accionar didáctico mucho más directo, cuyo único fin es el desarrollo integral de la personalidad de los educandos.

Para este autor, alcanzar un nivel de comprensión adecuado por parte de los profesores, de todas estas cuestiones inherentes al proceso de enseñanzaaprendizaje, resulta de suma importancia porque le permiten entender cómo opera el proceso en el orden teórico, a partir de su caracterización, propone que el proceso de enseñanza-aprendizaje es multidimensional, a partir de tres dimensiones: la humana, la técnica, como acción intencional, sistemática, que procura organizar las condiciones que propician mejor el aprendizaje y la dimensión político-social al considerar que el proceso de enseñanza y aprendizaje es situado ya que acontece en una cultura específica, trata con personas concretas que tienen una posición de clase definida en la organización social en que viven.

Al respecto, González (2002) plantea que existen dudas y problemas que afectan el desempeño profesional del maestro, al dirigir el proceso de enseñanza y aprendizaje, no se sabe aplicar en la práctica los elementos teóricos que caracterizan dicho proceso. Lo teórico se ve muy alejado de la práctica educativa y por tanto no se le brinda la importancia y el interés adecuado en tanto, teoría y práctica, constituyen una unidad dialéctica. Se habla de la clase como si fuera lo mismo que el Proceso de Enseñanza $y$ Aprendizaje y no sólo una parte de este, se reduce el todo a una de sus partes. El proceso de enseñanza y aprendizaje se centra en el profesor, sin atender a los procesos de aprendizaje que ocurren en los / las aprendices. Estos se consideran sujetos pasivos, reproductivos, a los que no se ofrecen oportunidades para la reflexión y el cuestionamiento; los docentes se anticipan a los juicios y análisis de los escolares y no los implican en la búsqueda de sus conocimientos, lo que frena el desarrollo de su pensamiento.

Asimismo, los contenidos curriculares carecen en ocasiones de pertinencia $y$ significatividad individual y social, y se ofrecen a partir de la lógica de las disciplinas independientes, de forma aislada, desconectada. El proceso tiene un carácter eminentemente reproductivo, tradicionalista, esquemático; lo instructivo y cognitivo es separado de lo afectivo y lo educativo, entendiéndose que las actividades educativas corresponden a las tareas Extra docentes y extraescolares, no a la clase.

Allport (2008) al referirse a la complejidad de este proceso, expone que aprendemos a andar, a hablar, a bailar; a recordar hechos, a interpretar números $\mathrm{y}$ recitar poemas. Aprendemos lo que conviene comer, lo que se debe tomar, lo que es preciso evitar, qué objetos son deseables sexualmente. Se adoptan religiones, creencias, ideologías. Se forman preferencias, prejuicios, modos de comportamiento. Aprendemos conceptos, significados y hábitos nuevos; también aprendemos lenguas extranjeras. Aprendemos a conocer signos, claves y símbolos. Adquirimos gradualmente nuestros rasgos $y$ orientaciones de la 
personalidad y desarrollamos una conciencia personal guiadora y una filosofía más o menos completa. Incluso aprendemos a aprender.

Se hace menester conocer la opinión de algunos autores respecto al aprendizaje. Castellanos (2005) define el aprendizaje humano como el proceso dialéctico de apropiación de los contenidos y las formas de conocer, hacer y convivir y son construidos en la experiencia socio histórica, en el cual se producen, como resultado de la actividad del individuo de la interacción con otras personas, cambios relativamente duraderos $\mathrm{y}$ generalizables, que le permiten adaptarse a la realidad, transformarla y crecer como personalidad.

Para Bermúdez (2004) aprender es un proceso dinámico: es el cambio que se produce en los conocimientos y estructuras mentales mediante la experiencia interactiva de los mismos y de lo que llega de afuera del individuo. El aprendizaje se acumula de modo que pueda servir como guía en el futuro y base de otros aprendizajes.

Pozo, (2008) expresa que de todas las definiciones de aprendizaje, tanto en la cultura popular como en la producción científica, se pueden extraer tres rasgos prototípicos del buen aprender: un cambio duradero y transferible a nuevas situaciones como consecuencia directa de la práctica realizada.

Es importante destacar que una de las cosas que influye considerablemente en el aprendizaje es la interacción con el medio, con los demás individuos, estos elementos modifican la forma de analizar y apropiarse de la información. A través del aprendizaje un individuo puede adaptarse al entorno y responder frente a los cambios y acciones que se desarrollan a su alrededor, cambiando si es esto necesario para subsistir. Para poder lograr los objetivos educativos, establecidos en los programas escolares, los profesores planifican, organizan, gestionan e implementan en el aula el proceso de enseñanza y aprendizaje $\mathrm{y}$ esto lo hacen según su formación, experiencia y recursos con que cuentan en el contexto donde laboran. La orquestación del proceso de enseñanza no es una tarea sencilla, demanda del docente conocimiento teórico y conocimiento práctico, habilidades cognitivas $\mathrm{y}$ sociales, destrezas, actitudes y valores deseables, así como una buena dosis de intuición o sentido común, entre otras.

Actualmente las prácticas pedagógicas apuntan a un aprendizaje centrado en el estudiante y por tanto, se presenta un cambio en el papel del docente ya no es el que se dedica a transmitir conocimientos, a instruir, sino orientar el proceso educativo en ambientes en los cuales el estudiante sea capaz de identificar lo que va aprender y las condiciones de cómo hacerlo en otras palabras se le está dando mayor importancia al aprendizaje autónomo donde el estudiante será capaz de identificar sus necesidades de aprendizaje y de acudir a las fuentes de información y procesos de formación para satisfacer esas necesidades. Este tipo de aprendizaje se viene aplicando ampliamente en procesos de instrucción relacionados con la formación profesional en diversas disciplinas, el desarrollo de recursos humanos en la empresa, la educación continuada, etc.

Según Piqueras (2014) cada persona aprende de una forma totalmente distinta de aprender. En cada persona existe una tendencia innata sobre la forma de integrar, asimilar y adquirir conocimientos. Los estilos de aprendizaje tienen que ver con nuestro enfoque hacia la forma en la que mejor aprendemos. Se recomienda que para maximizar nuestra forma de aprender comprendamos los distintos estilos de aprendizaje del resto de personas a las que formamos y el nuestro propio. 
Becerra y otros (2012), en su libro Aprender a Aprender, plantea que cuando un estudiante ingresa a la universidad debe desarrollar determinadas habilidades básicas $\mathrm{y}$ entre ellas propone: organizar y planificar su tiempo de estudio, determinar lo esencial en un contenido, leer con rapidez $y$ profundidad, comunicarse con eficiencia y evaluar continuamente su desempeño como estudiante.

Woolfolk, (2006) ha explicado el término motivación atendiendo a los rasgos personales o características individuales de la persona. Algunas explicaciones consideran factores internos y personales como las necesidades e intereses de los individuos, en tanto que otras establecen factores externos como la presión, social, el castigo o las recompensas, entre otros. A partir del enfoque clásico se realiza una distinción entre motivación intrínseca y extrínseca. La autora es del criterio que debe estar presente la dialéctica de lo extrínseco y de lo intrínseco en el proceso de aprendizaje, aunque desde el desarrollo del aprendizaje autónomo debe existir un tránsito hacia el predominio de motivos intrínsecos, es decir, el gusto por aprender.

Por ello, es necesario considerar que, si no existe motivación intrínseca en el estudiante, la motivación extrínseca se vuelve relativa, porque el docente puede planear la clase, seleccionar las estrategias de enseñanza que potencien el aprendizaje autónomo y encontrarse con un muro de indiferencia del estudiante; quien solo pide un cuestionario, que le permita en un futuro resolver el examen, pues esto le ha dado resultado a lo largo de su vida académica. Por ello, se debe estimular la motivación intrínseca, pues ese deseo de aprender moviliza al estudiante frente a las opciones presentadas por el docente, aunque no se puede obviar los casos en los que el estudiantado llega motivado y el docente con su insuficiente desempeño se encarga de truncar todo deseo de aprender.

El aprendizaje autónomo requiere que la persona identifique cómo aprende de mejor manera, cuáles son sus debilidades lo cual puede identificar al saber cuál es su estilo de aprendizaje. Unido a ello, debe tener interés por aprender y aquí entra la motivación, sin descuidar el hecho de saber qué estrategias de aprendizaje poner en marcha atendiendo a la naturaleza de los contenido. Por otra parte, cuando el docente identifica los estilos de aprendizaje, le resultará más fácil seleccionar las estrategias de enseñanza que fomenten los aprendizajes.

Con relación al aprendizaje autónomo, es importante retomar lo expuesto por Benítez (2008) para quien el aprendizaje autónomo es aquel donde cada persona aprende y se desarrolla de manera distinta $\mathrm{y}$ a ritmo diferente que otros estudiantes, se aplica o se experimenta el aprendizaje con la realidad, es muy importante desarrollar un aprendizaje autónomo pues la vida siempre está cambiando y algo nuevo que aprender siempre habrá; el estudiante desarrolla la habilidad o la capacidad de relacionar problemas por resolver, buscar la información necesaria, analizar, generar ideas, sacar conclusiones y establecer el nivel de logro de sus objetivos.

Según Rivas y Reinoso (2016) el aprendizaje autónomo conocido también como aprendizaje autodirigido es aquel en el cual el individuo toma la iniciativa en el diseño de sus experiencias de aprendizaje, la auto dirección en el aprendizaje es una combinación de fuerzas tanto internas como externas de la persona, estableciendo en el estudiante una responsabilidad mayor respecto al proceso enseñanza aprendizaje. Para estos autores el aprendizaje autónomo es una habilidad que facilita el aprendizaje tomando el control y dirigiendo sus procesos 
de pensamiento. El aprendizaje autónomo contribuye a que el alumno sea independiente y que auto gestione su práctica, es decir, que sea capaz de autorregular sus acciones para aprender y alcanzar determinadas metas en condiciones específicas en que el sujeto es el que dirige su proceso de aprendizaje mediante la coordinación fijación de objetivos y utilización de recursos necesarios para su aprendizaje.

El estudiante autónomo es aquel que lleva a cabo su proceso de aprendizaje solo, por tanto, conoce sus estilos de aprendizaje, así como las áreas en las que tiene fortalezas y debilidades para aprender, es autocritico, se autoevalúa auto motiva, toma decisiones y tiene iniciativa para realizar cambios al detectar posibles mejoras en sus trabajos.

El aprendizaje autónomo es organizado, responsable con la distribución y aprovechamiento del tiempo. El fundamento de la autonomía es la capacidad creciente del estudiante de "aprender a aprender", como resultado del conocimiento y asimilación de lo aprendido.

\section{CONCLUSIONES}

A partir de la revisión bibliográfica realizada se pudo establecer que el proceso enseñanza aprendizaje resulta ser ampliamente dinámico y complejo, que ante este proceso los maestros deben ser ampliamente creativos para motivar a los estudiantes y desarrollar en ellos habilidades relacionadas al aprendizaje autónomo, lo que permitirá que cuenten con habilidades para continuar su procesos de aprendizaje con herramientas prácticas pero que además estimulé el pensamiento crítico, creativo colaborativo y desarrollé las habilidades cognitivas y meta cognitivas del estudiante para ser creadores de conocimiento.

El diagnóstico realizado y que involucró varios métodos como la entrevista, encuesta, revisión documental, revelaron que los estudiantes de primer semestre de la carrera de derecho de la Universidad Privada Domingo Savio requieren apoyo para el desarrollo del aprendizaje autónomo, se evidencia el desconocimiento de herramientas que les faciliten encaminar su proceso con mayor independencia y fomentar el pensamiento crítico.

La Estrategia didáctica para el desarrollo del aprendizaje autónomo está sustentada en fundamentos teóricos que sirvieron como base orientadora para su elaboración, aplicación y evaluación, cuyos resultados a partir del Criterio de especialistas, indican la validez teórica y viabilidad de la Estrategia didáctica para el desarrollo del aprendizaje autónomo. La Estrategia didáctica para el desarrollo del aprendizaje autónomo propuesta está conformada por acciones didácticas orientadas a trabajar elementos del aprendizaje autónomo mismas que posibiliten el intercambio de información entre los participantes del proceso de enseñanza aprendizaje.

Se considera necesario continuar profundizando los sustentos teóricos $\mathrm{y}$ metodológicos para lograr el desarrollo del aprendizaje autónomo en los estudiantes bajo la guía docente para lograr la autonomía en la educación superior, para mejorar la calidad de enseñanza en los estudiantes y docentes, lo mismo que continuar investigando sobre el proceso enseñanza- aprendizaje tomar en cuenta que el contexto del aprendizaje autónomo es importante la noción de la investigación científica, precisando que la investigación no solo es la acumulación de información sino la creación de conocimiento, a partir del aprendizaje autónomo.

Además, se pudo concluir, la necesidad de incluir la estrategia didáctica para el desarrollo del aprendizaje autónomo como una línea de trabajo metodológico para los 
estudiantes de la Universidad Privada Domingo Savio de Potosí, y aplicar esta estrategia didáctica en otros contactos universitarios, con la finalidad de perfeccionarla y validarla como una vía para el desarrollo del aprendizaje autónomo en diferentes contextos.

\section{REFERENCIAS}

Allport, G. (2008). La personalidad. Su configuración y desarrollo. Barcelona, España. Ed. Herder

Bermúdez, R. (2004). Aprendizaje formativo y crecimiento personal. La Habana, Cuba. Editorial Pueblo y Educación

Becerra, M.J. y coautores (2012). Aprender a Aprender. La Habana Editorial Félix Varela

Benítez G. (2008). Definición de aprendizajeautónomo. Recuperado de http://georginabenitez.blogspot.com/20 08/10/definicion-de-aprendizajeautonomo.htm

Brenes, O. (2003) Estrategias didácticas. Costa Rica. Universidad de Costa Rica

Candau, V. (2017). "Estado actual de la didáctica". Brasil Editora Vozes Ltda ARRIOLA (2001). Orientaciones para planificar el aprendizaje

Castellanos, D. (2005). Aprender y enseñar en la escuela. Una concepción desarrolladora. La Habana, Cuba. Editorial Pueblo y
Educación

Feo, R. (2009). Diseño de estrategias didácticas orientaciones básicas. Málaga, España Ediciones Aljibe

González, A.M. (2002). El proceso de enseñanza-aprendizaje iagente del cambio educativo? En: A. M. González y C Reinoso. Nociones de Sociología, Psicología y Pedagogía (pp.156-157). Editorial Pueblo y Educación. La Habana, Cuba

Piqueras, C. (2014). Los cuatro estilos de aprendizaje de Honey y Mumford. Recuperado de: https://www.cesarpiqueras.com/loscuatro-estilos-de-aprendizaje-de-honeyy-mumford/

Pozo J.I. (2008). Aprendices y Maestros. La nueva cultura del aprendizaje. Madrid. España. Alianza Editorial POZO J.I. (2006). Teorías cognitivas del aprendizaje. Ediciones, Morata. Madrid. España

Rivas, L. y Reinoso, C. (2016). Aprendizaje autónomo: un reto en la formación profesional del estudiante. Revista Órbita. La Habana, Cuba

Uria, L. (2001). Estrategias didácticoorganizativas para mejorar los centros educativos

Woolfolk, A. (2006). Psicología Educativa. México Editorial Person 